\title{
ON THE INITIAL-VALUE PROBLEM FOR COMPRESSIBLE FLUID FLOWS WITH VANISHING VISCOSITY
}

\author{
By BuI AN TON
}

\section{Introduction.}

It is the aim of this paper to establish the existence of a unique local solution of the Cauchy problem for the fundamental system of equations describing the motion of viscous compressible heat conducting fluids. The solution obtained is analytic in the space-variables and converges in an appropriate sense to the solution of the limiting system as the viscosity tends to zero. We shall approximate the equations by a system of nonlinear equations of Sobolev-Galpern type. Estimates, in the quasi-norms of the generalized Gevrey spaces of Leray and Ohya are obtained by comparing solutions of some simple ordinary differential equations.

Let $u$ be a vector-function and let $\rho, \theta$ be scalar functions. Consider the initial-value problem

$$
\left\{\begin{array}{l}
\rho_{\varepsilon}\left(\frac{\partial u_{\varepsilon}}{\partial t}+u_{\varepsilon} \cdot \nabla u_{\varepsilon}\right)+\operatorname{grad}\left(\rho_{\varepsilon}+\theta_{\varepsilon}\right)-\varepsilon A u_{\varepsilon}=0, \\
\rho_{\varepsilon} \theta_{\varepsilon}\left(\frac{\partial \theta_{\varepsilon}}{\partial t}+u_{\varepsilon} \cdot \operatorname{grad} \theta_{\varepsilon}-\rho_{\varepsilon} \cdot \operatorname{div} u_{\varepsilon}\right)-\chi \Delta \theta_{\varepsilon}-\varepsilon B u_{\varepsilon}=0, \\
\frac{\partial \rho_{\varepsilon}}{\partial t}+\operatorname{div}\left(u_{\varepsilon} \rho_{\varepsilon}\right)=0, \quad \rho_{\varepsilon}(x, t) \text { and } \theta_{\varepsilon}(x, t)>0 \text { on }(0, T) \times R^{3}, \\
u_{\varepsilon}(x, 0)=u_{0}(x), \quad \rho_{\varepsilon}(x, 0)=\rho_{0}(x) \text { and } \theta_{\varepsilon}(x, 0)=\theta_{0}(x) \text { on } R^{3} .
\end{array}\right.
$$

$A$ is the linear elliptic operator $A u=\Delta u+\operatorname{grad}(\operatorname{div} u)$ and $B$ is the nonlinear operator $B u=\left(\partial u_{\imath} / \partial x_{k}+\partial u_{k} / \partial x_{\imath}\right)^{2}$ with the usual summation convention.

The equations describe the motion of compressible fluids with viscosity $\varepsilon$. The velocity, the density and the absolute temperature of the fluid are denoted by $u_{\varepsilon}, \rho_{\varepsilon}$ and $\theta_{\varepsilon}$ respectively. The coefflcient of heat conduction is $\chi$.

Unlike the case of incompressible fluids with constant density, there are few mathematical works on viscous compressible ones. In 1959, Serrin [9] proved a uniqueness theorem for solutions of (0.1) on bounded domains using the energy method. The problem of the existence of a local solution of (0.1)

Received February 20, 1979 
was first studied by Nash [6] in 1962 . He used a characteristic transformation, an iteration method and together with estimates for fundamental solutions of parabolic equations solved a nonlinear parabolic system at each step. In [10], Tani has casted some doubts on the validity of Nash's proof. During the last few years, Itaya [1] and Tani [10] have shown the existence of a unique local solution of (0.1). In both works, as done earlier by Nash, the characteristic transformation and estimates for fundamental solutions of a parabolic equation are used. Itaya applied the Tikhonov fixed point theorem where as Tani used the iteration method. The above approaches involve delicate computations.

The purpose of this paper is two-fold. We shall prove the existence of a unique local solution of (0.1), analytic in the space variables. We shall show that as the viscosity tends to zero, the solution of (0.1) will converge in an appropriate sense to the unique solution of the initial-value problem:

$$
\left\{\begin{array}{l}
\rho\left(\frac{\partial u}{\partial t}+u \cdot \nabla u\right)+\operatorname{grad}(\rho+\theta)=0, \\
\rho \theta\left(\frac{\partial \theta}{\partial t}+u \cdot \operatorname{grad} \theta-\rho \operatorname{div} u\right)-\chi \Delta \theta=0, \\
\frac{\partial \rho}{\partial t}+\operatorname{div}(u \rho)=0, \quad \rho(x, t) \text { and } \theta(x, t)>0 \text { on }(0, T) \times R^{3}, \\
u(x, 0)=u_{0}(x), \quad \rho(x, 0)=\rho_{0}(x) \text { and } \theta(x, 0)=\theta_{0}(x) \text { on } R^{3} .
\end{array}\right.
$$

The solution of (0.2) is analytic in the space variables.

The method used in this paper is different from that of Nash as well as that of Itaya. We shall approximate the solution of (0.1) by those of a system of nonlinear evolution equations of Sobolev-Galpern type. Estimates, in the quasi-norms of Leray and Ohya [2], for the approximating solutions are obtained by comparing solutions of a differential inequality with that of a differential equation.

The notations, simple propositions and the main results of the paper are given in Section 1. The existence of a local solution of a system of nonlinear evolution equations of Sobolev-Galpern type is proved in Section 2. Proofs of the main theorems are carried out in Section 3.

Section 1. Let $x=\left(x_{1}, x_{2}, x_{3}\right)$ and let $D_{j}=\partial / \partial x_{\jmath}$. For each triple $\alpha=\left(\alpha_{1}, \alpha_{2}, \alpha_{3}\right)$, of non-negative integers we write:

$$
D^{\alpha}=\prod_{\jmath=1}^{3} D_{\jmath \jmath}^{\alpha} \quad \text { with } \quad|\alpha|=\sum_{\jmath=1}^{3} \alpha_{\jmath} .
$$

The inner product and the norm in $H=L^{2}\left(R^{3}\right)$ are denoted by (.,.) and by $\|\cdot\|$ respectively. The Sobolev space

$$
H^{m}=\left\{u: u \text { in } H, D^{\alpha} u \text { in } H \text { for }|\alpha| \leqq m\right\}
$$


is a Hilbert space with the norm

$$
\|u\|_{m, 2}=\left\{\sum_{|\alpha| \leqq m}\left\|D^{\alpha} u\right\|^{2}\right\}^{1 / 2}
$$

and the usual inner product.

The following result of the Sobolev imbedding theorem will be used throughout the paper

$$
H^{2} \subset \mathrm{C}^{0, \gamma}\left(R^{3}\right) ; \quad 0<\gamma<1 / 2 \text { and } H^{1} \subset L^{4}\left(R^{3}\right) .
$$

The above natural injection mappings are all continuous and $C^{0, r}\left(R^{3}\right)$ is the space of Holder continuous functions on $R^{3}$ with exponent $\gamma$. It is known that $H^{2}$ is an algebra with respect to pointwise multiplication.

Let $\lambda>0$ and let $k$ be a positive integer. Set:

$$
\|u ; \lambda, k\|=\sum_{j=0}^{k} \frac{\lambda^{\jmath}}{j !} \sup _{|\alpha|=\jmath}\left\|D^{\alpha} u\right\|_{2,2} ; \quad|u ; \lambda, k|=\sum_{j=0}^{k} \frac{\lambda^{\jmath}}{j !} \sup _{|\alpha|=j}\left\|D^{\alpha} u\right\|_{C^{2, r_{(R 3)}}}
$$

whenever the right hand sides of (1.1) are defined. It is not difflcult to check that $\|\cdot ; \lambda, k\|$ and $|\cdot ; \lambda, k|$ are quasi-norms. We shall write:

$$
\|u ; \lambda\|=\|u ; \lambda, \infty\| \text { and }|u ; \lambda|=|u ; \lambda, \infty| .
$$

Proposition 1.1. 1) Suppose that $\| u$; $\lambda\|\|$ and $\|v ; \lambda\|$ are finite for some $\lambda>0$, then:

(i) $\|u v ; \lambda\|\|C\| u ; \lambda\|\|\|v ; \lambda\| \|$,

(ii) $\frac{d^{\jmath}}{d \lambda^{\jmath}}\|u ; \lambda\| \leqq C\left(\lambda-\lambda_{1}\right)^{-j}\|u ; \lambda\|$ for some $0<\lambda_{1}<\lambda$,

2) Suppose that $\|u ; \lambda, k\|$ and $\|v ; \lambda, k\|$ are finite for some $\lambda>0$ and for $a$ finite $k$, then: $\|u v ; \lambda, k\| \leqq C\|u ; \lambda, k\|\|v ; \lambda, k\| . \quad C$ is independent of $k$.

Proof. The first part of the assertion has been proved by Leray and Ohya [3], p. 108-109. With $j=1$, we have by using the definition of derivative:

$$
0<\frac{d}{d \lambda}\|u ; \lambda\| \leqq \varepsilon+\left(\|u ; \lambda\|-\left\|u ; \lambda_{1}\right\|\right)\left(\lambda-\lambda_{1}\right)^{-1}
$$

for $0<\lambda-\lambda_{1}<\eta(\varepsilon)$.

Since $\left\|u ; \lambda_{1}\right\|$ is positive,

$$
\frac{d}{d \lambda}\|u ; \lambda\| \leqq \varepsilon+\|u ; \lambda\| /\left(\lambda-\lambda_{1}\right) \leqq C\|u ; \lambda\| /\left(\lambda-\lambda_{1}\right)
$$

for $0<\lambda-\lambda_{1}<\eta(\varepsilon)$.

Repeated applications of the argument give (ii). The last assertion is shown in exactly the same way except that we stop at $k$ instead of going to infinity.

Proposition 1.2. Suppose that $\|u ; \lambda, k\|$ and $|v ; \lambda, k|$ are finite. Then: 
ON THE INITIAL-VALUE PROBLEM FOR COMPRESSIBLE FLUID FLOWS

$$
\|u v ; \lambda, k\| \leqq C\|u ; \lambda, k\||v ; \lambda, k| .
$$

If \|\|$u$; $\lambda|| \mid$ and $|v ; \lambda|$ are finite for some $\lambda>0$, then

$$
\|\| v ; \lambda\|\leqq C\| u ; \lambda \||v ; \lambda| \text {. }
$$

Proof. The proof is almost identical to [3], p. 108-109. We have only to make some obvious changes.

Proposition 1.3. Let $S$ be a subset of $R$ and let $F$ be a mapping of $R^{3} \times S$ into R. Suppose that:

$$
\sum_{j, k=0}^{\infty} \frac{\lambda^{\jmath}}{\jmath !}-\frac{\mu^{k}}{k !} \sup _{|\alpha|=\jmath}\left\|D_{x}^{\alpha} D_{y}^{k} F\right\|_{C^{2,} \gamma_{(R 3 \times S)}}
$$

for $0<\lambda, \mu$.

If $|v ; \lambda|$ is finite for some $\lambda>0$ and $v$ is a mapping of $R^{3}$ into $S$, then:

$$
\begin{aligned}
& |F(\cdot, v) ; \lambda| \\
& \leqq C\left(1+\|v\|_{\left.C^{2, r_{(R 3)}}\right)^{3}} \sum_{j, k=0} \frac{\lambda^{\jmath}}{\jmath !} \frac{1}{k !}(|v ; \lambda|-|v ; 0|)^{k} \sup _{|\alpha|=j}\left\|D_{x}^{\alpha} D_{y}^{k} F\right\|_{C^{2,}, r_{(R 3 \times S)}} .\right.
\end{aligned}
$$

Proof. Cf. Nalimov [7]. A detailed proof with some obvious changes may also be found in [4], p. 148-150.

Remark. The proposition is still valid if $|v ; \lambda|$ is replaced by $|v ; \lambda, k|$. It suffices to replace $|F(\cdot, v) ; \lambda|$ by $|F(\cdot, v) ; \lambda, k|$ and the summation in (1.4) is taken up to $k$ only.

$L^{2}\left(0, T ; H^{m}\right)$ is the space of equivalence classes of functions $u(\cdot, t)$ from $(0, T)$ to $H^{m}$ which are $L^{2}$-integrable over $(0, T)$. It is a Hilbert space with the norm

$$
\left.\|u\|={ }_{L^{2}(0, T ; H}\right)=\left\{\int_{0}^{T}\|u(\cdot, t)\|_{m, 2}^{2} d t\right\}^{1 / 2}
$$

and the obvious inner product.

$L^{\infty}\left(0, T ; H^{m}\right)$ is similarly defined with the usual modification. The derivative of $u$ with respect to $t$ is denoted by $\partial u / \partial t$ or simply by $u^{\prime}$ when there is no confusion possible. The following notion of fractional time-derivative of Lions will be needed as a technical device in the proofs of the theorems.

Definition. Let $u$ be in $L^{2}\left(0, T ; H^{m}\right)$. Then $u$ is said to have a fractional time derivative $D_{t}^{r} u$ of order $\gamma>0$ if there exists $U$ in $L^{2}\left(-\infty,+\infty ; H^{m}\right)$ such that:

(i) $u(\cdot, t)=U(\cdot, t)$ a.e. on $(0, T)$,

(ii) $\tau^{\gamma} \hat{U}(\cdot, \tau)$ is in $L^{2}\left(-\infty,+\infty ; H^{m}\right)$.

$\hat{U}(\cdot, \tau)$ is the Fourier transform of $U$ with respect to $t$.

We shall now state the main results of the paper. 
THEOREM 1.1. Let $u_{0}$ be a vector-function with $\left\|u_{0} ; \lambda\right\|$ finite for some $\lambda>0$. Let $\rho_{0}$ and $\theta_{0}$ be scalar functions and suppose that

(i) $\min \left(\right.$ g. ${ }_{R^{3}}$ b. $\rho_{0}$, g. ${ }_{R^{3}}$ b. $\left.\theta_{0}\right) \geqq c>0$,

(ii) $\left\|\operatorname{grad} \rho_{0} ; \lambda\right\|\|+\| \operatorname{grad} \theta_{0} ; \lambda \|+\left|\rho_{0} ; \lambda\right|+\left|\theta_{0} ; \lambda\right|<\infty$ for all $\lambda$ with $0<\lambda<\lambda_{0}$,

(iii) $\sum_{\jmath, k=0}^{\infty} \frac{\lambda^{\jmath}}{\jmath !} \frac{\mu^{k}}{k !}\left(\sup _{|\alpha|=\jmath}\left\|D_{x}^{\alpha} D_{y}^{k}\left(\rho_{0}+y\right)^{-1}\right\|_{C^{2, \gamma}(R 3 \times S)}+\sup _{|\alpha|=\jmath}\left\|D_{x}^{\alpha} D_{y}^{k}\left(\theta_{0}+y\right)^{-1}\right\|_{C^{\left.2, \gamma_{(R 3} \times S\right)}}\right)$ is finite for $\lambda, \mu>0$.

$S$ is the set $\{y:-\nu c<y<\infty ; 0<\nu<1\}$.

Then there exist.

(1) a non-empty interval $\left(0, T_{*}\right)$,

(2) a unique solution $\left\{u_{\varepsilon}, \rho_{\varepsilon}^{*}, \theta_{\varepsilon}^{*}\right\}$ of the inttial-value problem (0.1) on $\left(0, T_{*}\right) \times R^{3}$.

Moreover

$$
\left\|u_{\varepsilon}(\cdot, t) ; \lambda\right\|+\left\|\rho_{\varepsilon}^{*}(\cdot, t)-\rho_{0} ; \lambda\right\|+\left\|\theta_{\varepsilon}^{*}(\cdot, t)-\theta_{0} ; \lambda\right\| \leqq C
$$

on $\left(0, T_{*}\right)$ for all $\lambda$ with $0<\lambda<\lambda_{*}<\lambda_{0}$.

$C$ and $T_{*}$ are independent of $\varepsilon$.

For inviscid fluids, we have:

THEOREM 1.2. Suppose all the hypotheses of Theorem 1.1 are satisfied. Then there is a unique solution $\left\{u, \rho^{*}, \theta^{*}\right\}$ of the initial-value problem $(0.2)$ on $\left(0, T_{*}\right)$ $\times R^{3}$. Moreover

$$
\left\|u_{\varepsilon}(\cdot, t)-u(\cdot, t) ; \lambda\right\|\|+\| \rho_{\varepsilon}^{*}(\cdot, t)-\rho^{*}(\cdot, t) ; \lambda\|+\| \theta_{\varepsilon}^{*}(\cdot, t)-\theta^{*}(\cdot, t) ; \lambda \| \rightarrow 0
$$

uniformly on $\left(0, T_{*}\right)$ as $\varepsilon \rightarrow 0 . \quad\left\{u_{\varepsilon}, \rho_{\varepsilon}^{*}, \theta_{\varepsilon}^{*}\right\}$ and $\left(0, T_{*}\right)$ are as in Theorem. 1.1.

Section 2. In this section we shall prove the existence of a local solution of the following initial-value problem:

$$
\left\{\begin{array}{l}
\rho^{*}(u+\eta L u)^{\prime}+\rho^{*}(u \cdot \nabla u)+\operatorname{grad}\left(\rho^{*}+\theta^{*}\right)-\varepsilon A u=0, \quad 0<\eta<\varepsilon<1, \\
\rho^{*} \theta^{*}\left(\theta^{*}+\eta L \theta^{*}\right)^{\prime}+\rho^{*} \theta^{*}\left(u \cdot \operatorname{grad} \theta^{*}-\rho^{*} \operatorname{div} u\right)-\chi \Delta \theta^{*}-\varepsilon B u=0, \\
\left(\rho^{*}+\eta L \rho^{*}\right)^{\prime}+\operatorname{div}\left(u \rho^{*}\right)=0 ; \rho^{*} \text { and } \theta^{*}>0 \quad \text { on }(0, T) \times R^{3}, \\
u(x, 0)=u_{0}(x), \quad \rho^{*}(x, 0)=\rho_{0}(x) \text { and } \theta^{*}(x, 0)=\theta_{0}(x) \text { on } R^{3} .
\end{array}\right.
$$

$L$ is the linear elliptic operator defined by:

$$
L=\sum_{|\alpha|,|\beta| \leqq 4}(-1)^{|\alpha|} D^{\alpha} D^{\beta} .
$$

The main result of this section is the following theorem.

THEOREM 2.1. Suppose all the hypotheses of Theorem 1.1 are satisfied. Then there exist: 
(i) a non-empty interval $\left(0, T_{*}\right)$ independent of both $\varepsilon$ and $\eta$,

(ii) a solution $\left\{u, \rho^{*}, \theta^{*}\right\}$ of the inttral-value problem (2.1).

Moreover for some $\lambda>0$ :

$$
\|u(\cdot, t) ; \lambda\|\|+\| \rho^{*}(\cdot, t)-\rho_{0}(\cdot) ; \lambda\|+\| \theta^{*}(\cdot, t)-\theta_{0}(\cdot) ; \lambda \| \leqq C
$$

on $\left(0, T_{*}\right) . \quad C$ is a constant independent of both $\varepsilon$ and $\eta$.

The proof of the theorem is long. We shall outline the main steps of the proof before going into the details.

Step. 1. The Galerkin approximation method is used to show the existence of a local solution of the following system of nonlinear evolution equations of Sobolev-Galpern type :

$$
\left\{\begin{array}{l}
(u+\eta L u)^{\prime}+u \cdot \nabla u+\left(\operatorname{grad}\left(\rho+\rho_{0}\right)+\operatorname{grad}\left(\theta+\theta_{0}\right)-\varepsilon A u\right) / \rho_{*}=0, \\
(\theta+\eta L \theta)^{\prime}+u \cdot \operatorname{grad}\left(\theta+\theta_{0}\right)-\left(\rho+\rho_{0}\right) \operatorname{div} u-\left(\chi \Delta\left(\theta+\theta_{0}\right)+\varepsilon B u\right) / \rho_{*} \theta_{*}=0 \\
(\rho+\eta L \rho)^{\prime}+\operatorname{div} u\left(\rho+\rho_{0}\right)=0 \text { on }(0, T) \times R^{3} \\
u(x, 0)=u_{0}(x), \quad \rho(x, 0)=0=\theta(x, 0) \text { on } R^{3} .
\end{array}\right.
$$

$\rho_{*}=\rho+\rho_{0}+\left\|(\rho+\nu c)^{-}\right\|_{L^{\infty}(R 3)}$ and similarly for $\theta_{*}$. We denoted by $(\rho+\nu c)^{-}$ the expression:

$(\rho+\nu c)^{-}=0$ if $\rho+\nu c \geqq 0$ and is equal to $-(\rho+\nu c)$ if $(\rho+\nu c)<0$.

Step 2. From the result of the previous step, we deduce the existence of a non-empty interval $\left(0, T_{n}^{*}\right)$ where $(\rho+\nu c)^{-}=(\theta+\nu c)^{-}=0$ and hence $\rho+\rho_{0}$ as well as $\theta+\theta_{0}$ are non-negative on $\left(0, T^{*}\right) \times R^{3}$. Using the difference quotient method and then the quasi-norms of Leray and Ohya, it will be shown that

$$
\|u(\cdot, t) ; \lambda\|+\|\rho(\cdot, t) ; \lambda\|+\|\theta(\cdot, t) ; \lambda\| \leqq C(\lambda) \quad \text { on }\left(0, T_{\eta}^{*}\right) .
$$

It is in establishing the above estimate that we need the perturbed equations of Sobolev-Galpern type.

Step. 3. Once $u, \rho$ and $\theta$ are known to be in the generalized Gevrey spaces, we may apply part (ii) of Proposition 1.1. Using the technique of comparing solutions of a differential inequality with a solution of a differential equation we show that there exist a non-empty interval $\left(0, T_{*}\right)$ and a constant $C$ such that

$$
\|u(\cdot, t) ; \lambda\|+\|\rho(\cdot, t) ; \lambda\|+\|\theta(\cdot, t) ; \lambda\| \leqq C \quad \text { on }\left(0, T_{*}\right) .
$$

$C$ and $T_{*}$ are independent of both $\varepsilon$ and $\eta$.

We shall now proceed to the proof of the existence of a local solution of (2.3).

Let $\left\{w_{j}\right\}$ be a vector-function basis of the separable Hilbert space $H^{4}$. For each $j$, there exists a unique $v_{j}$ in $H^{8}$ such that: 


$$
L v_{j}=w_{\jmath} .
$$

Since $\left\{w_{j}\right\}$ is a basis of the Hilbert space $H^{4}$, it is not difficult to check that $\left\{v_{j}\right\}$ is a basis of $H^{8}$.

Let $\varphi$, be a scalar function basis of the separable Hilbert space $H^{4}$ of scalar functions. Again for each $\jmath$, there exists a unique $\phi_{\jmath}$ in $H^{8}$ such that:

$$
L \phi_{j}=\varphi_{j} .
$$

$\left\{\phi_{j}\right\}$ is a basis of $H^{8}$. Set:

$$
u_{k}=\sum_{j=1}^{k} c_{j k}(t) v_{\jmath} ; \quad \rho_{k}=\sum_{j=1}^{k} d_{j k}(t) \psi_{\jmath} \quad \text { and } \quad \theta_{k}=\sum_{j=1}^{k} e_{j k}(t) \psi_{\jmath} .
$$

Since $u_{0}$ is in $H^{8}$, there exist real numbers $\alpha_{j k}$ such that:

$$
\sum_{j=1}^{k} \alpha_{j k} v_{j} \rightarrow u_{0} \text { in } H^{8} .
$$

Consider the system of nonlinear ordinary differential equations in $\left\{c_{j_{k}}, d_{j k}, e_{j k}\right\}$ :

$$
\left\{\begin{aligned}
&\left(\left(u_{k}+\eta L u_{k}\right)^{\prime}, w_{j}\right)+\left(u_{k} \cdot \nabla u_{k}, w_{\jmath}\right)+\left(\left\{\operatorname{grad}\left(\rho_{k}+\rho_{0}\right)+\operatorname{grad}\left(\theta_{k}+\theta_{0}\right)\right\} / \rho_{* k}, w_{\jmath}\right) \\
&-\varepsilon\left(A u_{k} / \rho_{* k}, w_{j}\right)=0, \\
&\left(\left(\theta_{k}+\eta L \theta_{k}\right)^{\prime}, \varphi_{j}\right)+\left(u_{k} \cdot \operatorname{grad}\left(\theta_{k}+\theta_{0}\right)-\left(\rho_{k}+\rho_{0}\right) \operatorname{div} u_{k}, \varphi_{\jmath}\right)-\left(\left[\chi \Delta\left(\theta_{k}+\theta_{0}\right)+\varepsilon B u_{k}\right] / \rho_{* k} \theta_{* k}, \varphi_{j}\right)=0, \\
&\left(\left(\rho_{k}+\eta L \rho_{k}\right)^{\prime}, \varphi_{j}\right)+\left(\operatorname{div} u_{k}\left(\rho_{k}+\rho_{0}\right), \varphi_{j}\right)=0, \quad 1 \leqq \jmath \leqq k, \\
& c_{j k}(0)=\alpha_{j k}, \quad d_{j k}(0)=e_{j k}(0)=0 \quad \text { with } \\
& \rho_{* k}=\rho_{k}+\rho_{0}+\left\|\left(\rho_{k}+\nu c\right)^{-}\right\|_{L^{\infty}\left(R^{3}\right)} \quad \text { and similarly for } \theta_{* k} .
\end{aligned}\right.
$$

LEMma 2.1. Suppose all the hypotheses of Theorem 1.1 are satisfied. Then there exists a local solution in $C\left(0, T_{s \eta}^{k} ; H^{8}\right)$ of the system (2.7) depending on $k, \varepsilon, \eta$ and which we write as $u_{k}, \rho_{k}, \theta_{k}$. Moreover:

$$
\rho_{k}+\rho_{0} \text { and } \theta_{k}+\theta_{0} \geqq(1-\nu) c \text { on }\left(0, T_{\varepsilon \eta}^{k}\right) \times R^{3} \text {. }
$$

Proof. 1) We note that

$$
\left(\left(u_{k}+\eta L u_{k}\right)^{\prime}, w_{\jmath}\right)=\sum_{s=1}^{k} c_{s k}^{\prime}(t)\left(v_{s}+\eta L v_{s}, w_{j}\right)=\sum_{s=1}^{k} c_{s k}^{\prime}\left(v_{s}+\eta L v_{s}, L v_{j}\right)
$$

by applying (2.4). Since $\left\{v_{j}\right\}$ is a basis of $H^{8}$, $\operatorname{det}\left\{\left(v_{s}, v_{j}\right)+\eta\left(L v_{s}, L v_{j}\right)\right\}$ is nonzero. Indeed, let

$$
[v, w]=(v, w)+\eta(L v, L w) \text { for } v, w \text { in } H^{8} .
$$

Then the inner product $[\cdot, \cdot]$ induces a norm which is equivalent to the $H^{8}$ norm. Since $\left\{v_{j}\right\}$ is a basis of $H^{8}$, $\operatorname{det}\left[v_{s}, v_{\jmath}\right] \neq 0$. Similarly for $\operatorname{det}\left\{\left(\phi_{s}, \phi_{j}\right)+\right.$ 
$\left.\eta\left(L \psi_{s}, L \psi_{j}\right)\right\}$.

It follows from the Caratheodory theorem that there exists a local solution of $(2.7)$ in $C\left(0, T_{\varepsilon \eta}^{k *} ; H^{8}\right)$. Since $\rho_{k}$ and $\theta_{k}$ are in $C\left(0, T_{\varepsilon \eta}^{k *} ; H^{8}\right)$ and $\rho_{k}(x, 0)$ $=\theta_{k}(x, 0)=0$, there exists a non-empty interval $\left(0, T_{s \eta n}^{k}\right)$ such that :

$$
\left|\rho_{k}(x, t)\right|, \quad\left|\theta_{k}(x, t)\right| \leqq c \nu \quad \text { on }\left(0, T_{\varepsilon \eta \eta}^{k}\right) \times R^{3} .
$$

Let $\left(\rho_{k}+\nu c\right)^{-}$be as before and $\left(\rho_{k}+\nu c\right)^{+}=\rho_{k}+\nu c$ if $\rho_{k}+\nu c \geqq 0$ and 0 otherwise. Then :

$$
\rho_{k}+\rho_{0}=\left(\rho_{k}+\nu c\right)^{+}-\left(\rho_{k}+\nu c\right)^{-}+\rho_{0}-\nu c \geqq(1-\nu) c
$$

on $\left(0, T_{\varepsilon \eta}^{k}\right) \times R^{3}$. Similarly for $\theta_{k}+\theta_{0}$.

LEMMA 2.2. Let $\left\{u_{k}, \rho_{k}, \theta_{k}\right\}$ be as in Lemma 2.1. Then there exist:

(1) a non-empty interval $\left(0, T_{\eta}\right)$,

(2) a constant $C_{\eta}$ such that

$$
\left\|u_{k}\right\|_{L^{\infty}\left(0, T_{\eta}, H^{8)}\right.}+\left\|\rho_{k}\right\|_{L^{\infty}\left(0, T_{\eta}, H^{8)}\right.}+\left\|\theta_{k}\right\|_{L^{\infty}\left(0, T_{\eta}, H^{8)}\right.} \leqq C_{\eta} .
$$

Moreover: $\rho_{k}+\rho_{0}, \theta_{k}+\theta_{0} \geqq(1-\nu)$ c on $\left(0, T_{\eta}\right) \times R^{3} . T_{\eta}$ and $C_{\eta}$ are independent of $k$ and $\varepsilon$.

Proof. 1) We multiply the first equation of (2.7) by $c_{j k}$ then take the summation with respect to $j$ from 1 to $k$. Taking (2.4) and (2.6) into account we obtain :

Thus,

$$
\begin{array}{r}
\left(\frac{d}{d t}\left(u_{k}+\eta L u_{k}\right), L u_{k}\right)+\left(u_{k} \cdot \nabla u_{k}, L u_{k}\right)+\left(\left\{\operatorname{grad}\left(\rho_{k}+\rho_{0}\right)\right.\right. \\
\left.\left.+\operatorname{grad}\left(\theta_{k}+\theta_{0}\right)\right\} / \rho_{* k}, L u_{k}\right)-\varepsilon\left(A u_{k} / \rho_{* k}, L u_{k}\right)=0
\end{array}
$$

$$
\begin{aligned}
& \frac{d}{d t}\left(\left\|u_{k}\right\|_{4,2}^{2}+\eta\left\|L u_{k}\right\|^{2}\right) \leqq 2\left\|L u_{k}\right\|\left(\left\|u_{k} \cdot \nabla u_{k}\right\|+\left\|\operatorname{grad}\left(\rho_{k}+\rho_{0}\right) / \rho_{*_{k}}\right\|\right. \\
& \left.+\left\|\operatorname{grad}\left(\theta_{k}+\theta_{0}\right) / \rho_{* k}\right\|+\left\|A u_{k} / \rho_{* k}\right\|\right) \\
& \leqq 2\left\|L u_{k}\right\|\left(C_{\eta}\left\|u_{k}\right\|_{4,2}^{2}+\left\|\rho_{k}\right\|_{1,2}+\left\|\theta_{k}\right\|_{1,2}+\left\|u_{k}\right\|_{2,2}+1\right) .
\end{aligned}
$$

$C_{\eta}$ depends only on $\eta$ but is independent of both $k$ ahd $\varepsilon$. In the above estimate we have applied the Sobolev imbedding theorem. Hence:

$$
\frac{d}{d t}\left(\left\|u_{k}\right\|_{4,2}^{2}+\eta\left\|L u_{k}\right\|^{2}\right) \leqq C_{\eta}\left(1+\eta\left\|L u_{k}\right\|^{2}+\left\|u_{k}\right\|_{4,2}^{4}+\left\|\rho_{k}\right\|_{4,2}^{2}+\left\|\theta_{k}\right\|_{4,2}^{2}\right) .
$$

2) With the second and third equations of (2.7) we obtain by a similar argument :

$$
\begin{aligned}
& \frac{d}{d t}\left(\left\|\rho_{k}\right\|_{4,2}^{2}+\left\|\theta_{k}\right\|_{4,2}^{2}+\eta\left\|L \rho_{k}\right\|^{2}+\eta\left\|L \theta_{k}\right\|^{2}\right) \\
& \quad \leqq C_{\eta}\left(1+\left\|\rho_{k}\right\|_{4,2}^{4}+\left\|\theta_{k}\right\|_{4,2}^{4}+\left\|u_{k}\right\|_{4,2}^{4}+\eta\left\|L \rho_{k}\right\|^{2}+\eta\left\|L \theta_{k}\right\|^{2}\right) .
\end{aligned}
$$


The different constants $C_{\eta}$ are all independent of $k$ and of $\varepsilon$.

From (2.8)-(2.9) we have:

$$
\frac{d}{d t} E_{k}(t) \leqq C\left(1+E_{k}^{2}(t)\right) ; \quad E_{k}(0) \leqq\left\|u_{0}\right\|_{4,2}^{2}+\left\|L u_{0}\right\|^{2}
$$

with

$$
\begin{aligned}
E_{k}(t)= & \left\|u_{k}(t)\right\|_{4,2}^{2}+\left\|\rho_{k}(t)\right\|_{4,2}^{2}+\left\|\theta_{k}(t)\right\|_{4,2}^{2} \\
& +\eta\left(\left\|L u_{k}(t)\right\|^{2}+\left\|L \rho_{k}(t)\right\|^{2}+\left\|L \theta_{k}(t)\right\|^{2}\right) .
\end{aligned}
$$

3) Consider the initial-value problem

$$
\frac{d x}{d t}=C_{\eta}\left(1+x^{2}\right), \quad x(0)=\left\|u_{0}\right\|_{4,2}^{2}+\left\|L u_{0}\right\|_{4,2}^{2} .
$$

It is known that (2.11) has a solution $x_{\eta}$ in $C\left(0, T_{\eta}^{*}\right)$ and clearly $T_{\eta}^{*}$ is independent of $k$ and of $\varepsilon$. A standard comparison theorem of the theory of ordinary differential equations applied to (2.10)-(2.11) yields:

$$
E_{k}(t) \leqq x_{\eta}(t) \text { on } \min \left(\left[0, T_{\eta}^{*}\right],\left[0, T_{\varepsilon \eta}^{k}\right]\right) \text {. }
$$

4) From (2.9) and the above estimate we obtain by using the Sobolev imbedding theorem

$$
\left\|\rho_{k}(\cdot, t)\right\|_{L^{\infty}\left(R^{3}\right)} ; \quad\left\|\theta_{k}(\cdot, t)\right\|_{L^{\infty}\left(R^{3}\right)} \leqq C \nu
$$

on $\min \left(\left[0, T_{\varepsilon \eta}^{k}\right],\left[0, T_{\eta}\right]\right)$ with $T_{\eta}=\min \left(T_{\eta}^{*}, c^{2} \nu^{2} / C_{\eta}^{2}\right)$.

By continuation if necessary we have:

The lemma is proved.

$$
E_{k}(t) \leqq x_{\eta}(t) \leqq C_{\eta} \quad \text { on }\left[0, T_{\eta}\right] .
$$

LeMmA 2.3. Let $u_{k}, \rho_{k}, \theta_{k}$ be as in Lemma 2.1. Then:

$$
\left\|D_{t}^{r} u_{k}\right\|_{L^{2}\left(0, T_{\eta}, H\right)}+\left\|D_{t}^{r} \rho_{k}\right\|_{L^{2}\left(0, T_{\eta}, H\right)}+\left\|D_{t}^{r} \theta_{k}\right\|_{L^{2}\left(0, T_{\eta}, H\right)} \leqq C_{\eta} .
$$

$T_{\eta}$ is as in Lemma 2.2. $\gamma$ us any number with $0<\gamma<1 / 4$ and $C_{\eta}$ is independent of $k$ and of $\varepsilon$.

Proof. 1) Set $\tilde{u}_{k}(\cdot, t)=u_{k}(\cdot, t)$ on $\left[0, T_{\eta}\right]$ and zero otherwise. Similarly for $\tilde{\rho}_{k}$ and for $\tilde{\theta}_{k}$. The first equation of (2.7) may be rewritten as:

$$
\begin{aligned}
\left(\frac{d}{d t}\left(\tilde{u}_{k}+\eta L \tilde{u}_{k}\right), w_{\jmath}\right)+\left(\tilde{u}_{k} \cdot \nabla \tilde{u}_{k}, w_{\jmath}\right)+\left(\left\{\operatorname{grad}\left(\rho_{k}+\rho_{0}\right)\right\} \sim, w_{j} / \rho_{* k}\right) \\
\quad+\left(\left\{\operatorname{grad}\left(\theta_{k}+\theta_{0}\right)\right\} \sim / \rho_{* k}, w_{j}\right)-\varepsilon\left(A \tilde{u}_{k} / \rho_{* k}, w_{\jmath}\right) \\
=\delta(0)\left(u_{k}(0)+\eta L u_{k}(0), w_{j}\right)-\delta\left(T_{\eta}\right)\left(u_{k}\left(T_{\eta}\right)+\eta L u_{k}\left(T_{\eta}\right), w_{j}\right) .
\end{aligned}
$$

$\delta(0)$ and $\delta\left(T_{\eta}\right)$ are the Dirac delta functions with mass at 0 and at $T_{\eta}$ respec- 
tively.

Taking the Fourier transform of (2.12) with respect to $t$ we obtain:

$$
\begin{aligned}
i \tau\left(\left(\hat{u}_{k}+\eta L \hat{u}_{k}\right), w_{\jmath}\right)+\left(\left(\tilde{u}_{k} \cdot \nabla \tilde{u}_{k}\right)^{\wedge}, w_{j}\right)+\left(\left\{\left(\operatorname{grad}\left(\rho_{k}+\rho_{0}\right)\right)^{\sim} / \rho_{* k}\right\}^{\wedge}, w_{\jmath}\right) \\
\quad+\left(\left\{\left(\operatorname{grad}\left(\theta_{k}+\theta_{0}\right)\right)^{\sim} / \rho_{* k}\right\}^{\wedge}, w_{\jmath}\right)-\varepsilon\left(\left(A \tilde{u}_{k} / \rho_{* k}\right)^{\wedge}, w_{\jmath}\right) \\
=\left(u_{k}(0)+\eta L u_{k}(0), w_{j}\right)-\exp \left(2 i T_{\eta} \pi\right)\left(u_{k}\left(T_{\eta}\right)+\eta L u_{k}\left(T_{\eta}\right), w_{\jmath}\right) .
\end{aligned}
$$

$\hat{u}_{k}$ is the Fourier transform of $\tilde{u}$ with respect to $t$.

Using (2.6) and applying Lemma 2.2, we get by an elementary computation as in the first part of the proof of Lemma 2.1 :

$$
\begin{aligned}
& |\tau|\left\|\hat{u}_{k}\right\|_{4,2}^{2} \leqq\left\|L \hat{u}_{k}\right\|\left(C_{\eta}+\left\|\left(A \tilde{u}_{k} / \rho_{* k}\right)^{\wedge}\right\|+\left\|\left(\tilde{u}_{k} \cdot \nabla \tilde{u}_{k}\right)^{\wedge}\right\|\right. \\
& \left.\quad+\left\|\left\{\left(\operatorname{grad}\left(\rho_{k}+\rho_{0}\right)\right)^{\sim} / \rho_{* k}\right\}^{\wedge}\right\|+\left\|\left\{\left(\operatorname{grad}\left(\theta_{k}+\theta_{0}\right)\right)^{\sim} / \rho_{*_{k}}\right\}^{\wedge}\right\|\right) .
\end{aligned}
$$

An application of the Sobolev imbedding theorem and of Lemma 2.2 yields:

$$
\begin{aligned}
& \left\|\left(A \tilde{u}_{k} / \rho_{* k}\right)^{\wedge}\right\|+\left\|\left(\tilde{u}_{k} \cdot \nabla \tilde{u}_{k}\right)^{\wedge}\right\|+\left\|\left\{\left(\operatorname{grad}\left(\rho_{k}+\rho_{0}\right)\right)^{\sim} / \rho_{* k}\right\} \wedge\right\| \\
& \quad+\left\|\left\{\left(\operatorname{grad}\left(\theta_{k}+\theta_{0}\right)\right)^{\sim} / \rho_{* k}\right\}^{\wedge}\right\| \\
& \leqq \\
& \quad(1-\nu)^{-1} c^{-1} \int_{0}^{T \eta}\left(\left\|u_{k}\right\|_{2,2}+\left\|\rho_{k}\right\|_{2,2}+\left\|\theta_{k}\right\|_{2,2}+\left\|u_{k}\right\|_{3,2}^{2}\right) d t \leqq C .
\end{aligned}
$$

Thus,

$$
|\tau|\left\|\hat{u}_{k}\right\|_{4,2}^{2} \leqq C_{\eta}\left\|L \hat{u}_{k}\right\| \text {. }
$$

The different constants $C_{\eta}$ are all independent of $k$ and of $\varepsilon$. Let $\sigma$ be any number with $2 \sigma>1$. Then applying Lemma 2.2 we obtain

$$
\int_{-\infty}^{\infty}\left(1+|\tau|^{\sigma}\right)^{-1}\left\|\hat{u}_{k}\right\|_{1,2}^{2} d \tau \leqq C_{\eta}
$$

An elementary argument as in Lions [5], p. 79 gives:

$$
\left\|D_{t}^{r} u_{k}\right\|_{L^{2}\left(0, T_{\eta}, H\right)} \leqq C_{\eta} .
$$

$0<\gamma<1 / 4$ and $C_{\eta}$ is independent of both $k$ and $\varepsilon$.

An argument exactly as above with the second and third equations of (2.7) yields :

$$
\left\|D_{t}^{\gamma} \rho_{k}\right\|_{L^{2}\left(0, T_{\eta}, H\right)}+\left\|D_{t}^{r} \theta_{k}\right\|_{L^{2}\left(0, T_{\eta}, H\right)} \leqq C_{\eta} .
$$

The lemma is proved.

LEMMA 2.4. Let $\left\{u_{0}, \rho_{0}, \theta_{0}\right\}$ be as in Theorem 1.1. Then there exist:

1) a non-empty interval $\left(0, T_{\eta}\right)$,

2) a solution $\left\{u_{\varepsilon \eta}, \rho_{\varepsilon \eta}^{*}, \theta_{\varepsilon \eta}^{*}\right\}$ which we write as $\left\{u, \rho^{*}, \theta^{*}\right\}$ of the initial-value problem (2.1). 
Moreover: $\rho=\rho^{*}-\rho_{0}, \theta=\theta^{*}-\theta_{0}$ and $u$ are in $L^{\infty}\left(0, T_{\eta} ; H^{8}\right)$.

Proof. 1) Let $\left\{u_{k}, \rho_{k}, \theta_{k}\right\}$ be as in Lemmas 2.1-2.3. From the estimates of the above lemmas we have by taking subsequences if necessary: $u_{k}-u$ in the weak*-topology of $L^{\infty}\left(0, T_{\eta} ; H^{8}\right), D_{t}^{r} u_{k} \rightarrow D_{t}^{r} u$ weakly in $L^{2}\left(0, T_{\eta} ; H\right)$ as $k \rightarrow+\infty$. Similarly for $\rho_{k}$ and for $\theta_{k}$.

Let $G$ be any bounded open subset of $R^{3}$. From the estimates of Lemmas 2.2 and 2.3 we get:

$$
\left\|u_{k}\right\|_{L^{\infty}\left(0, T_{\eta}, H 8(G)\right)}+\left\|D_{t}^{\gamma} u_{k}\right\|_{L^{2\left(0, T_{\eta}, H(G)\right)} \leqq C_{\eta}} .
$$

$C_{\eta}$ is independent of $k$.

The natural injection mapping of $H^{8}(G)$ into $H^{7}(G)$ is compact. It follows from (2.17) and from a theorem of Lions [5], p. 61 that: $u_{k} \rightarrow u$ in $L^{p}\left(0, T_{\eta}\right.$; $H^{\eta}(G)$ ) and a. e. on $\left(0, T_{\eta}\right) \times G$ for $2 \leqq p<\infty$. Similarly $\rho_{k} \rightarrow \rho$ and $\theta_{k} \rightarrow \theta$ in $L^{p}\left(0, T_{\eta} ; H^{7}(G)\right)$ and a. e. on $\left(0, T_{\eta}\right) \times G$ as $k \rightarrow+\infty$.

2) Applying the Sobolev imbedding theorem and Lemma 2.2 we obtain:

$$
\begin{aligned}
\| u_{k} & \cdot \nabla u_{k}\left\|_{L^{2}\left(0, T_{\eta}, H\right)}+\right\| \operatorname{div}\left(u_{k} \cdot \rho_{k}\right)\left\|_{L^{2}\left(0, T_{\eta}, H\right)}+\right\| B u_{k} \|_{L^{2}\left(0, T_{\eta}, H\right)} \\
& +\left\|A u_{k} / \rho_{* k}\right\|_{L^{2}\left(0, T_{\eta}, H\right)}+\left\|\operatorname{grad}\left(\rho_{k}+\rho_{0}\right) / \rho_{* k}\right\|_{\left.L^{2(0, T}, T_{\eta}, H\right)} \\
& +\left\|B u_{k} / \rho_{*_{k}} \theta_{*_{k}}\right\|_{\left.L^{2(0}, T_{\eta}, H\right)}+\left\|\operatorname{grad}\left(\theta_{k}+\theta_{0}\right) / \rho_{*_{k}}\right\|_{\left.L^{2(0, T}, T_{\eta}, H\right) \leqq C_{\eta}} .
\end{aligned}
$$

$C_{\eta}$ is independent of $k$.

We now show that the above expressions converge to their appropriate limits as $k \rightarrow+\infty$. It suffices to show that $B u_{k} / \rho_{*_{k}} \theta_{*_{k}} \rightarrow B u / \rho_{*} \theta_{*}$ weakly in $L^{2}\left(0, T_{\eta} ; H\right)$. Identical arguments will give the desired results.

From the weak compactness of the unit ball in a Hilbert space we have by taking subsequences if necessary: $B u_{k} / \rho_{* k} \theta_{* k} \rightarrow f$ weakly in $L^{2}\left(0, T_{\eta} ; H\right)$. It is clear that $\rho_{* k}=\rho_{k}+\rho_{0} \rightarrow \rho_{*}=\rho+\rho_{0} \geqq(1-\nu) c$ a. e. on $\left(0, T_{\eta}\right) \times G$ and $\theta_{* k}=$ $\theta_{k}+\theta_{0} \rightarrow \theta_{*}=\theta+\theta_{0} \geqq(1-\nu) c$ a. e. on $\left(0, T_{\eta}\right) \times G$. An application of the Lebesgue convergence theorem yields:

$$
B u_{k} / \rho_{* k} \theta_{* k} \rightarrow B u / \rho_{*} \theta_{*} \text { weakly in } L^{2}\left(0, T_{\eta} ; H(G)\right) .
$$

$H(G)$ denotes $L^{2}(G)$. Hence: $f=B u / \rho_{*} \theta_{*}$ a. e. on $\left(0, T_{\eta}\right) \times G$. Since $f$ and $B u / \rho_{*} \theta_{*}$ are both in $L^{2}\left(0, T_{\eta} ; H\right)$ and $G$ is any bounded open subset of $R^{3}$, we have: $f=B u / \rho_{*} \theta_{*}$ a. e on $\left(0, T_{\eta}\right) \times R^{3}$.

3) It now follows from the above and from standard arguments of the theory of partial differential equations that $\left\{u, \rho^{*}=\rho+\rho_{0}, \theta^{*}=\theta+\theta_{0}\right\}$ is a solution of the initial-value problem (2.1).

The lemma is proved and thus, the first step of the proof of Theorem 2.1 as outlined earlier at the beginning of the section has been carried out.

We shall now show that

$$
\|u(t) ; \lambda\|+\|\rho(t) ; \lambda\|+\|\theta(t) ; \lambda\| \leqq C_{\eta} \text { on }\left(0, T_{\eta}\right) \text { for all } 0<\lambda<\lambda_{0} .
$$


It is step 2 of the proof of Theorem 2.1.

LemmA 2.5. Let $\left\{u, \rho=\rho^{*}-\rho_{0}, \theta=\theta^{*}-\theta_{0}\right\}$ be as in Lemma 2.4. Then there exist a non-empty interval $\left(0, T_{\eta}^{*}\right)$ and a constant $C_{\eta}$ such that:

$$
\|u(t) ; \lambda\|+\|\rho(t) ; \lambda\|+\|\theta(t) ; \lambda\| \leqq C_{\eta} \quad \text { on }\left(0, T_{\eta}^{*}\right)
$$

for all small $\lambda, 0<\lambda<\lambda_{0}$. The constants $T_{\eta}^{*}$ and $C_{\eta}$ are independent of $\varepsilon$.

Proof. 1) From Lemma 2.4 we have:

$$
\left\{\begin{array}{l}
(u+\eta L u)^{\prime}=\varepsilon A u / \rho^{*}-u \cdot \nabla u-\operatorname{grad}\left(\rho^{*}\right) / \rho^{*}-\operatorname{grad}\left(\theta^{*}\right) / \theta^{*}, \\
(\theta+\eta L \theta)^{\prime}=-u \cdot \operatorname{grad}\left(\theta^{*}\right)+\rho^{*} \operatorname{div} u-\left(\chi \Delta \theta^{*}+\varepsilon B u\right) / \rho^{*} \theta^{*}, \\
(\rho+\eta L \rho)^{\prime}=-\operatorname{div}\left(u \rho^{*}\right) \text { on }\left(0, T_{\eta}\right) \times R^{3}, \\
\rho(x, 0)=\theta(x, 0)=0 \text { and } u(x, 0)=u_{0}(x) \text { on } R^{3} .
\end{array}\right.
$$

Since $u, \rho$ and $\theta$ are in $L^{\infty}\left(0, T_{\eta} ; H^{8}\right)$ it follows from the Sobolev imbedding theorem and from our hypotheses on $\rho_{0}, \theta_{0}$ that the right hand side of the first equation of (2.18) is in $L^{\infty}\left(0, T_{\eta} ; H^{1}\right)$. The method of difference quotient applied to the first equation of $(2.18)$ yields $u$ in $L^{\infty}\left(0, T_{\eta} ; H^{9}\right)$. Similarly, $\rho$ and $\theta$ are in $L^{\infty}\left(0, T_{\eta} ; H^{9}\right)$. Now the right hand side of the first equation of (2.18) is in $L^{\infty}\left(0, T_{\eta} ; H^{2}\right)$ and thus, $u$ is in $L^{\infty}\left(0, T_{\eta} ; H^{10}\right)$. Repeating the bootstrap argument we get $\{u, \rho, \theta\}$ in $L^{\infty}\left(0, T_{\eta} ; H^{s}\right)$ for all $s>0$.

2) From (2.18) we have by an elementary argument:

for all $\alpha$.

$$
\begin{gathered}
\frac{d}{d t}\left(\left\|D^{\alpha} u\right\|_{2,2}^{2}+\eta\left\|D^{\alpha} u\right\|_{1,2}^{2}\right)^{1 / 2} \leqq\left\|D^{\alpha}(u \cdot \nabla u)\right\|_{2,2}+\left\|D^{\alpha}\left(A u / \rho^{*}\right)\right\|_{2,2} \\
+\left\|D^{\alpha}\left(\operatorname{grad}\left(\rho^{*}\right) / \rho^{*}\right)\right\|_{2,2}+\left\|D^{\alpha}\left(\operatorname{grad}\left(\theta^{*}\right) / \rho^{*}\right)\right\|_{2,2}
\end{gathered}
$$

Let $\lambda>0$. It follows from the above inequality, from Propositions 1.1.-1.3 and from the definition of the quasi-norms $\|\cdot ; \lambda, s\|$ and $|\cdot ; \lambda, s|$ that

$$
\begin{aligned}
& E_{s}(u(t) ; \lambda) \leqq C\left\|u_{0} ; \lambda\right\|+C \int_{0}^{t}(\|u ; \lambda, s\|\|\| \nabla u ; \lambda, s \|+1+ \\
& \quad\left|\left(\rho+\rho_{0}\right)^{-1} ; \lambda, s\right|(\|\operatorname{grad} \rho ; \lambda, s\|+\|\operatorname{grad} \theta ; \lambda, s\|+\|\Delta u ; \lambda, s\|) d t .
\end{aligned}
$$

$E_{s}(u(t) ; \lambda)$ is the expression $\|u(t) ; \lambda, s\|+\eta^{1 / 2}\|\nabla u(t) ; \lambda, s\|+\eta^{1 / 2}\|\Delta u(t) ; \lambda, s\|$. The constant $C$ is independent of $s$.

Applying Proposition 1.3 and the remark following it, we obtain:

$$
\begin{aligned}
& E_{s}(u(t) ; \lambda) \leqq C\left(\left\|u_{0} ; \lambda\right\|+\int_{0}^{t}(1+\|u ; \lambda, s\|\|\nabla u ; \lambda, s\|\right. \\
& \quad+\{\|\operatorname{grad} \rho ; \lambda, s\|+\|\operatorname{grad} \theta ; \lambda, s\|+\|\Delta u ; \lambda, s\|\}\}(|\rho ; \lambda, s|)) d t .
\end{aligned}
$$

$G(r)$ is a positive continuously increasing function of its argument. It is the 
function defined in (1.3) with $r=\mu$ and $F=\left(y+\rho_{0}\right)^{-1}$.

An application of the Sobolev imbedding theorem gives:

$$
|\rho ; \lambda, s| \leqq C(\|\rho ; \lambda, s\|+\|\operatorname{grad} \rho ; \lambda, s\|+\|\Delta \rho ; \lambda, s\|) .
$$

In view of (2.21), the inequality (2.20) becomes :

$$
E_{s}(u(t) ; \lambda) \leqq C\left(\left\|u_{0} ; \lambda\right\|+\int_{0}^{t} C_{\eta}\left(E_{s}^{2}(u ; \lambda)+E_{s}^{2}(\rho ; \lambda)+E_{s}^{2}(\theta ; \lambda)+1\right) d t .\right.
$$

The different constants $C_{\eta}$ are all independent of $s, t$ and $\varepsilon$.

2) From the second and the third equation of (2.18) we get by similar arguments :

$$
E_{s}(\rho(t) ; \lambda)+E_{s}(\theta(t) ; \lambda) \leqq C_{\eta} \int_{0}^{t}\left(1+\left\{E_{s}(\rho ; \lambda)+E_{s}(\theta ; \lambda)+E_{s}(u ; \lambda)\right.\right.
$$

Combining (2.22)-(2.23) we obtain :

$$
\left.\left.+G^{2}(\rho ; \lambda)\right\} G^{2}\left(E_{s}(\theta) ; \lambda\right)\right) d t .
$$

$$
R_{s}(t ; \lambda) \leqq C\left\|u_{0} ; \lambda\right\|+C_{\eta} \int_{0}^{t}\left(1+R_{s}^{2}(z)+G^{2}\left(R_{s}(z)\right)+G^{4}\left(R_{s}(z)\right)\right) d z
$$

with

$$
R_{s}(t)=E_{s}(u(t) ; \lambda)+E_{s}(\rho(t) ; \lambda)+E_{s}(\theta(t) ; \lambda) .
$$

3) Consider the integral equation:

$$
x(t)=C\left\|u_{0} ; \lambda\right\|+C_{\eta} \int_{0}^{t}\left(1+x^{2}+G^{2}(x)+G^{4}(x)\right) d t .
$$

It is clear that the integral equation (2.25) has a local solution $x_{\eta}(t)$ in $C\left(0, T_{\eta}^{*}\right)$ and obviously $T_{\eta}^{*}$ does not depend on $s$. A standard comparison theorem applied to (2.24)-(2.25) yields :

$$
R_{s}(t) \leqq x_{\eta}(t) \text { on } \min \left(\left[0, T_{\eta}\right],\left[0, T_{\eta}^{*}\right]\right) \text {. }
$$

Let $s \rightarrow+\infty$ and we get the lemma by taking into account the definition of $R_{s}(t)$ and of $E_{s}(u(t) ; \lambda)$.

We now carry out the last step as outlined at the end of Theorem 2.1.

Proof of Theorem 2.1. 1) From Lemma 2.5 we know that $\|u(t) ; \lambda\|$, $\|\rho(t) ; \lambda\|$ and $\|\theta(t) ; \lambda\|$ are finite for some small $\lambda>0$ and for $t$ in $\left[0, T_{\eta}^{*}\right]$.

Thus, in the inequality (2.19) we have:

$$
\begin{gathered}
\|\| u(t) ; \lambda\|\leqq\| u_{0} ; \lambda\|\|+\int_{0}^{t}(\|u ; \lambda\|\|\| \nabla u ; \lambda \|+\{\|\operatorname{grad} \rho ; \lambda\|+\|\operatorname{grad} \theta ; \lambda\| \\
\left.+\|\Delta u ; \lambda\|\}\left|\left(\rho+\rho_{0}\right)^{-1} ; \lambda\right|\right) d t .
\end{gathered}
$$

Applying Proposition 1.1 we get: 
ON THE INITIAL-VALUE PROBLEM FOR COMPRESSIBLE FLUID FLOWS

$$
\begin{aligned}
\|u(t) ; \lambda\| \leqq\left\|u_{0} ; \lambda\right\|+C\left(\lambda-\lambda_{1}\right)^{-2} \int_{0}^{t}\left(\|u ; \lambda\|^{2}+\{\|\rho ; \lambda\|+\|\theta ; \lambda\| \|\right. \\
\left.+\|u ; \lambda\| \|\}\left|\left(\rho+\rho_{0}\right)^{-1} ; \lambda\right|\right) d t . \\
\leqq\left\|u_{0} ; \lambda\right\|+C\left(\lambda-\lambda_{1}\right)^{-2} \int_{0}^{t}\left(1+\|u ; \lambda\|^{2}+\|\theta ; \lambda\|^{2}\right. \\
\left.+\|\rho ; \lambda\|^{2}+G^{2}(|\rho ; \lambda|)\right) d t .
\end{aligned}
$$

$G$ is a positive continuously increasing function of its argument as in the proof of Lemma 2.5 .

Similarly from (2.24) we have:

$$
\begin{aligned}
\|\rho ; \lambda\|+\|\theta ; \lambda\| \leqq C\left(\lambda-\lambda_{1}\right)^{-2} \int_{0}^{t}\left(1+\|u ; \lambda\|^{2}+\|\rho ; \lambda\|^{2}+\|\theta ; \lambda\|^{2}\right. \\
\left.+G^{4}(\|\rho ; \lambda\|+\|\theta ; \lambda\| \|)\right) d t .
\end{aligned}
$$

The different constants $C$ are all independent of $t, \varepsilon$ and $\eta . \lambda-\lambda_{1}$ is small and strictly positive.

It follows from (2.26)-(2.27) that

$$
\begin{gathered}
\|u(t) ; \lambda\|+\|\rho(t) ; \lambda\|+\|\theta(t) ; \lambda\| \geqq\left\|u_{0} ; \lambda\right\| \\
+C \int_{0}^{t}\left(1+(\|u ; \lambda\|+\|\rho ; \lambda\|+\|\theta ; \lambda\|)^{2}\right. \\
\left.\quad+G^{4}(\|u ; \lambda\|+\|\rho ; \lambda\|+\|\theta ; \lambda\|)\right) d t .
\end{gathered}
$$

$C$ depends on $\lambda$, but is independent of $t, \varepsilon$ and $\eta$.

2) Consider the nonlinear integral equation:

$$
x(t)=\left\|u_{0} ; \lambda\right\|+C(\lambda) \int_{0}^{t}\left(1+x^{2}+G^{4}(x)\right) d t .
$$

Clearly (2.29) has a local solution $x(t)$ in $C\left(0, T^{*}\right)$ and obviously $T^{*}$ is independent of both $\varepsilon$ and $\eta$.

A standard comparison theorem applied to (2.28)-(2.29) gives:

$$
\|u(t) ; \lambda\|+\|\rho(t) ; \lambda\|+\|\theta(t) ; \lambda\| \leqq x(t) \quad \text { on } \min \left(\left[0, T_{n}^{*}\right],\left[0, T^{*}\right]\right) .
$$

From (2.29)-(2.30), we have:

$$
\|\rho(\cdot, t)\|_{2,2} ; \quad\|\theta(\cdot, t)\|_{2,2} \leqq C t \quad \text { on } \min \left(\left[0, T_{\eta}^{*}\right],\left[0, T^{*}\right]\right) \text {. }
$$

Since $\rho(\cdot, 0)=\theta(\cdot, 0)=0$, it follows from the Sobolev imbedding theorem that:

$$
\begin{aligned}
& \|u(t) ; \lambda\|+\|\rho(t) ; \lambda\|+\|\theta(t) ; \lambda\| \leqq C \text { and } \\
& \|\rho(\cdot, t)\|_{L^{\infty}\left(R^{3}\right)}, \quad\|\theta(\cdot, t)\|_{L^{\infty}\left(R^{3}\right)} \leqq c \nu \text { on } \min \left(\left[0, T_{\eta}^{*}\right],\left[0, T_{*}\right]\right)
\end{aligned}
$$

with $T_{*}=\min \left(T^{*}, \nu c / C\right)$. 
$C$ is independent of both $\varepsilon$ and $\eta$ but depends on $\lambda$.

By continuation if necessary we obtain:

$$
\begin{aligned}
& \|u(t) ; \lambda\|+\|\rho(t) ; \lambda\|+\|\theta(t) ; \lambda\| \leqq C \text { and } \\
& \|\rho(\cdot, t)\|_{L^{\infty}\left(R^{3}\right)}, \quad\|\theta(\cdot, t)\|_{L^{\infty}\left(R^{3}\right)} \leqq C \nu \text { on }\left[0, T_{*}\right] .
\end{aligned}
$$

Set : $\rho^{*}=\rho+\rho_{0}, \theta^{*}=\theta+\theta_{0}$ and we get the theorem.

Section 3. We shall now turn to the proofs of Theorems 1.1 and 1.2. First we need a technical lemma.

LEMMA 3.1. Let $\left\{u_{\varepsilon \eta}, \rho_{\varepsilon \eta}^{*}=\rho_{\varepsilon \eta}+\rho_{0}, \theta_{\varepsilon \eta}^{*}=\theta_{\varepsilon \eta}+\theta_{0}\right\}$, which we shall write as $\left\{u_{\eta}, \rho_{\eta}^{*}, \theta_{\eta}^{*}\right\}$ be as in Theorem 2.1. Then:

$$
\left\|D_{t}^{r} u_{\eta}\right\|_{L^{2\left(0, T_{*}, H\right)}}+\left\|D_{t}^{r} \rho_{\eta}\right\|_{L^{2\left(0, T_{*}, H\right)}}+\left\|D_{t}^{r} \theta_{\eta}\right\|_{L^{2\left(0, T_{*}, H\right)}} \leqq C .
$$

$0<\gamma<1 / 4$ and $C$ is a constant independent of both $\varepsilon$ and $\eta$.

Proof. The proof is exactly the same as that of Lemma 2.3 but by using the estimates of Theorem 2.1 instead of those of Lemma 2.2, the constant $C$ is independent of both $\varepsilon$ and $\eta$.

Proof of Theorem 1.1. 1) Let $\left\{u_{\eta}, \rho_{\eta}, \theta_{\eta}\right\}$ be as in Theorem 2.1. From the estimates of Theorem 2.1 we get by taking subsequences if necessary: $u_{\eta} \rightarrow u=u_{\varepsilon}$ in the weak*-topology of $L^{\infty}\left(0, T_{*} ; H^{s}\right)$ for all $s$ as $\eta \rightarrow 0$. Similarly for $\rho_{\eta}$ and for $\theta_{\eta}$. We have:

$$
\|u(t) ; \lambda\|+\|\rho(t) ; \lambda\|+\|\theta(t) ; \lambda\| \leqq C(\lambda) \quad \text { on }\left(0, T_{*}\right)
$$

for small positive $\lambda>0$.

$C$ is a constant independent of.

From Lemma 3.1, we obtain by taking subsequences:

$$
D_{t}^{r} u_{\eta} \rightarrow D_{t}^{r} u, \quad D_{t}^{r} \rho_{\eta} \rightarrow D_{t}^{r} \rho \quad \text { and } \quad D_{t}^{r} \theta_{\eta} \rightarrow D_{t}^{r} \theta \quad \text { weakly in } L^{2}\left(0, T_{*} ; H\right)
$$

as $\eta \rightarrow 0$. An argument as in part (2) of the proof of Lemma 2.4 yields :

$$
\left\{\begin{array}{l}
u^{\prime}+u \cdot \nabla u+\left(\operatorname{grad} \rho^{*}+\operatorname{grad} \theta^{*}-\varepsilon A u\right) / \rho^{*}=0, \\
\theta^{\prime}+u \cdot \operatorname{grad} \theta^{*}-\rho^{*} \operatorname{div} u-\left(\chi \Delta \theta^{*}+\varepsilon B u\right) / \rho^{*} \theta^{*}=0, \\
\rho^{\prime}+\operatorname{div}\left(u \rho^{*}\right)=0, \quad \rho^{*}=\rho+\rho_{0}, \theta^{*}=\theta+\theta_{0} \geqq(1-\nu) c \quad \text { on }\left(0, T_{*}\right) \times R^{3}, \\
u(x, 0)=u_{0}(x), \rho(x, 0)=\theta(x, 0)=0 \text { on } R^{3} .
\end{array}\right.
$$

2) It remains to show that the solution is unique. Suppose that $\left\{u_{1}, \rho_{1}^{*}, \theta_{1}^{*}\right\}$ and $\left\{u_{2}, \rho_{2}^{*}, \theta_{2}^{*}\right\}$ are two solutions of (3.1) with all the properties stated in the theorem. Set: $u=u_{1}-u_{2}, \rho=\rho_{1}-\rho_{2}$ and $\theta=\theta_{1}-\theta_{2}$. Then : 


$$
\left\{\begin{array}{c}
u^{\prime}+u_{1} \cdot \nabla u+u \cdot \nabla u_{2}+\operatorname{grad} \rho / \rho_{1}^{*}-\rho \operatorname{grad} \rho_{2}^{*} / \rho_{1}^{*} \rho_{2}^{*}+\operatorname{grad} \theta / \rho_{1}^{*} \\
-\rho \operatorname{grad} \theta_{2}^{*} / \rho_{1}^{*} \rho_{2}^{*}-\varepsilon \Delta u / \rho_{1}^{*}+\varepsilon \Delta u_{2} \cdot \rho / \rho_{1}^{*} \rho_{2}^{*}=0, \\
\theta^{\prime}+u \cdot \operatorname{grad} \theta_{1}+u_{2} \cdot \operatorname{grad} \theta_{2}-\rho \operatorname{div} u_{1}-\rho_{2}^{*} \operatorname{div} u-\varepsilon B u_{1} / \rho_{1}^{*} \theta_{1}^{*} \\
\quad+\varepsilon B u_{2} / \rho_{2}^{*} \theta_{2}^{*}-\chi \Delta \theta / \rho_{1} \theta_{1}+\chi \rho \Delta \theta_{2} / \rho_{1} \theta_{1} \rho_{2}+\chi \theta \Delta \theta_{2} / \theta_{1} \theta_{2} \rho_{2}=0, \\
\rho^{\prime}+\operatorname{div}\left(u_{1} \rho\right)+\operatorname{div}\left(u \rho_{2}\right)=0 \quad \text { on }\left(0, T_{*}\right) \times R^{3}, \\
u(x, 0)=\rho(x, 0)=\theta(x, 0)=0 \quad \text { on } R^{3} .
\end{array}\right.
$$

From the first equation of (3.2) we have for all small $\lambda$ with $0<\lambda<\lambda_{0}$

$$
\begin{aligned}
& \|u(t) ; \lambda\| \leqq \int_{0}^{t}\left(\left\|\Delta u / \rho_{1}^{*} ; \lambda\right\|+\left\|\rho \Delta u_{2} / \rho_{1}^{*} \rho_{2}^{*} ; \lambda\right\|+\left\|u_{1} \cdot \nabla u ; \lambda\right\| \|\right. \\
& \quad+\|\| u \cdot \nabla u_{2} ; \lambda\|+\| \operatorname{grad} \rho / \rho_{1}^{*} ; \lambda\|+\| \operatorname{grad} \theta / \rho_{1}^{*} ; \lambda\|\| \\
& \left.\quad+\left\|\rho \operatorname{grad} \rho_{2}^{*} / \rho_{1}^{*} \rho_{2}^{*} ; \lambda\right\|+\left\|\rho \operatorname{grad} \theta_{2}^{*} / \rho_{1}^{*} \rho_{2}^{*} ; \lambda\right\| \|\right) d t .
\end{aligned}
$$

Applying Propositions 1.1-1.3 and noting that $\left\|u_{\jmath} ; \lambda\right\|,\left|1 / \rho_{j}^{*} ; \lambda\right|$ as well as $\left|1 / \theta_{j}^{*} ; \lambda\right|$ are finite, we obtain:

$$
\|u(t) ; \lambda\| \leqq \int_{0}^{t} C(\lambda)(\|u(t) ; \lambda\|+\|\rho(t) ; \lambda\|+\|\theta(t) ; \lambda\|) d t .
$$

In exactly the same way we get from the third equation of (3.2):

$$
\|\| \rho(t) ; \lambda \| \leqq \int_{0}^{t} C(\lambda)(\|u(t) ; \lambda\|+\|\rho(t) ; \lambda\|+\|\theta(t) ; \lambda\|) d t .
$$

With the second equation of (3.2), we obtain by a similar argument after recalling the definition of $B$ in $(0.1)$ and using the elementary relation $a^{2}-b^{2}$ $=(a-b)(a+b)$,

$$
\|\theta(t) ; \lambda\| \geqq \int_{0}^{t} C(\lambda)(\|u(t) ; \lambda\|+\|\rho(t) ; \lambda\|+\|\theta(t) ; \lambda\|) d t .
$$

It follows from (3.3)-(3.5) that $u(x, t)=\rho(x, t)=\theta(x, t)=0$. The theorem is proved.

Proof of Theorem 1.2. 1) Let $\left\{u_{\varepsilon}, \rho_{\varepsilon}^{*}=\rho_{\varepsilon}+\rho_{0}, \theta_{\varepsilon}^{*}=\theta_{\varepsilon}+\theta_{0}\right\}$ be as in Theorem 1.1. From the estimates of Theorem 1.1 we have by taking subsequences if necessary : $u_{\varepsilon} \rightarrow u$ in the weak*-topology of $L^{\infty}\left(0, T_{*} ; H^{s}\right)$ for all $s$ as $\varepsilon \rightarrow 0$. Similarly for $\rho_{\varepsilon}$ and for $\theta_{\varepsilon}$. We have:

$$
\|u(t) ; \lambda\|+\|\rho(t) ; \lambda\|+\|\theta(t) ; \lambda\| \leqq C \quad \text { on }\left(0, T_{*}\right) .
$$

From the estimates of Lemma 3.1, we get : $D_{t}^{r} u_{\varepsilon} \rightarrow D_{t}^{r} u, D_{t}^{r} \rho_{\varepsilon} \rightarrow D_{t}^{r} \rho$ and $D_{t}^{r} \theta_{\varepsilon} \rightarrow D_{t}^{r} \theta$ weakly in $L^{2}\left(0, T_{*} ; H\right)$ as $\varepsilon \rightarrow 0$. Again a standard argument as in the proof of Lemma 2.4 shows that $\left\{u, \rho^{*}=\rho+\rho_{0}, \theta^{*}=\theta+\theta_{0}\right\}$ is a solution of (0.2). 
A proof as in part (2) of Theorem 1.1 shows that the solution is unique. Thus, we may take the sequence $\left\{u_{\varepsilon}, \rho_{\varepsilon}, \theta_{\varepsilon}\right\}$ instead of subsequences.

2) It remains to show that

$$
\left\|u_{\varepsilon}(t)-u(t) ; \lambda\right\|+\left\|\rho_{\varepsilon}(t)-\rho(t) ; \lambda\right\|+\left\|\theta_{\varepsilon}(t)-\theta(t) ; \lambda\right\| \rightarrow 0
$$

uniformly on $\left(0, T_{*}\right)$ as $\varepsilon \rightarrow 0$.

We have:

$$
\left\{\begin{array}{l}
\left(u_{\varepsilon}-u\right)^{\prime}+\left(u_{\varepsilon}-u\right) \cdot \nabla u+u \cdot \nabla\left(u_{\varepsilon}-u\right)+\operatorname{grad}\left(\rho_{\varepsilon}-\rho\right) / \rho_{\varepsilon}^{*}-\left(\rho_{\varepsilon}-\rho\right) \cdot \operatorname{grad} \rho^{*} / \rho_{\varepsilon}^{*} \rho^{*} \\
\quad+\operatorname{grad}\left(\theta_{\varepsilon}-\theta\right) / \rho_{\varepsilon}^{*}-\left(\rho_{\varepsilon}-\rho\right) \operatorname{grad} \theta^{*} / \rho^{*} \rho_{\varepsilon}^{*}-\varepsilon A u_{\varepsilon} / \rho_{\varepsilon}^{*}=0, \\
\left(\theta_{\varepsilon}-\theta\right)^{\prime}+\left(u_{\varepsilon}-u\right) \cdot \operatorname{grad} \theta_{\varepsilon}^{*}+u \cdot \operatorname{grad}\left(\theta_{\varepsilon}-\theta\right)-\left(\rho_{\varepsilon}-\rho\right) \operatorname{div} u_{\varepsilon}-\rho^{*} \operatorname{div}\left(u_{\varepsilon}-u\right) \\
\quad-\varepsilon B u_{\varepsilon} / \rho_{\varepsilon}^{*} \theta_{\varepsilon}^{*}=0, \\
\left(\rho_{\varepsilon}-\rho\right)^{\prime}+\operatorname{div} \rho\left(u_{\varepsilon}-u\right)+\operatorname{div}\left(u_{\varepsilon}\left(\rho_{\varepsilon}-\rho\right)\right)=0 \quad \text { on }\left(0, T_{*}\right) \times R^{3}, \\
u_{\varepsilon}(x, 0)-u(x, 0)=0, \quad \rho_{\varepsilon}(x, 0)-\rho(x, 0)=\theta_{\varepsilon}(x, 0)-\theta(x, 0)=0 \quad \text { on } R^{3} .
\end{array}\right.
$$

From the estimates of Theorem 1.1 we get:

$$
\left|1 / \rho_{\varepsilon}^{*} \theta_{\varepsilon}^{*} ; \lambda\right|+\left|1 / \rho^{*} \rho_{\varepsilon}^{*} ; \lambda\right|+\left|\left\|u_{\varepsilon} ; \lambda\right\|\right|+\mid\left\|\rho_{\varepsilon} ; \lambda\right\|+\left\|\theta_{\varepsilon} ; \lambda\right\| \leqq C
$$

for small positive $\lambda$ with $C$ independent of $\varepsilon$.

It follows from Propositions 1.1-1.3 and from (3.6) that:

$$
\begin{aligned}
& \left\|u_{\varepsilon}(t)-u(t) ; \lambda\right\|+\left\|\rho_{\varepsilon}(t)-\rho(t) ; \lambda\right\|+\left\|\theta_{\varepsilon}(t)-\theta(t) ; \lambda\right\| \\
& \quad \leqq C(\lambda) \int_{0}^{t}\left(\varepsilon+\left\|u_{\varepsilon}-u ; \lambda\right\|+\left\|\rho_{\varepsilon}-\rho ; \lambda\right\|+\left\|\theta_{\varepsilon}-\theta ; \lambda\right\|\right) d t .
\end{aligned}
$$

$C(\lambda)$ is independent of $\varepsilon$ and of $t$.

Applying the Gronwall lemma we get the theorem.

\section{REFERENCES}

[1] N. ITAYA: On the Cauchy problem for the system of fundamental equations describing the movement of compressible fluids. Kōdai Math. Sem. Report 23 (1971), 60-120.

[2] J. LERAY AND Y. OHyA: Equations et systèmes non lineáires, hyperboliques non stricts. Math. Annalen 170 (1967), 167-205.

[.3] J. Leray And Y. Ohya: Systemes linéaires, hyperboliques non stricts. Colloque Analyse Fonctionnelle (1964), 105-144. Centre Belge de Recherches Math.

[4] J. LeRAy AND L. WAElbroeck: Norme formelle d'une fonction composée. Colloque Analyse Fonctionnelle (1964), 145-152. Centre Belge de Recherches Math.

[5] J.L. Lions: Quelques méthodes de résolution des problèmes aux limites non linéaires Dunod. Paris (1970). 
ON THE INITIAL-VALUE PROBLEM FOR COMPRESSIBLE FLUID FLOWS

[6] J. NASH: Le problème de Cauchy pour les équations differentielles d'un fluide general. Bull. Soc. Math. France 90 (1962), 487-497.

[7] V.I. NALimov: A priori estimates of solutions of elliptic equations in the class of analytic functions and their applications to the Cauchy-Poisson problem. Sov. Math. Dokl. 10 (1969), 1350-1354.

[8] L.B. Ovsjannikov: Singular operator in the scale of Banach spaces. Sov. Math. Dokl. 6 (1965), 1025-1028.

[9] J. Serrin: On the uniqueness of compressible fluid motions. Arch. Rat. Mech. Anal. 3 (1959), 271-288.

[10] A. TANI: On the first initial boundary-value problem of compressible viscous fluid motion. Publi RIMS, Kyoto Univ. 13 (1977), 193-253.

[11] B. A. Ton: Nonlinear evolution equations of Sobolev-Galpern type. Math. $Z$. 151 (1976), 219-233.

Department of Mathematics, University of British Columbia, VANCOUVER-CANADA. 